Copyright by the American Physical Society. Rusjan, E.; Zweifel, P. F., "scattering kernels for the muon diffusion equation," Phys. Rev. A 38, 1860 (1988); DOI: http://dx.doi.org/10.1103/PhysRevA.38.1860

PHYSICAL REVIEW A

VOLUME 38, NUMBER 4

AUGUST 15, 1988

\title{
Scattering kernels for the muon diffusion equation
}

\author{
E. Rusjan and P. F. Zweifel \\ Center for Transport Theory and Mathematical Physics, Virginia Polytechnic Institute and State University, Blacksburg, Virginia 24061
}

(Received 11 January 1988)

\begin{abstract}
Diffusion of muonic hydrogen atoms in gaseous hydrogen is studied. Scattering kernels are derived from the kinematics of an inelastic binary collision. The effect of rotations of the hydrogen molecules is treated by defining and computing an effective inelastic energy transfer $Q_{\text {eff }}$. The Doppler effect is taken into account by averaging the cross sections over the Maxwellian velocity distribution of the target molecules.
\end{abstract}

\section{INTRODUCTION}

An understanding of the diffusion of muonic hydrogen atoms in molecular hydrogen is of interest both for the analysis of experiments on nuclear capture of muons ${ }^{1,2}$ and for the study of muon-catalyzed fusion. ${ }^{3-7}$

This diffusion process is governed by a time-, space-, and energy-dependent transport equation whose solution is intractable unless certain simplifying assumptions are made. We have begun an analysis of this diffusion process using an analog to the Selengut-Goertzel approximation of neutron transport theory. ${ }^{8}$ It is well known that muonic hydrogen atoms behave similarly to neutrons, because they are neutral and very small (Bohr radius approximately $250 \mathrm{fm}$ ). Hence the use of neutron transport theory is quite reasonable. In this approach the total scattering cross section for an atom of energy $E^{\prime}$ to scatter to energy $E$ through an angle $\cos ^{-1} \mu_{0}$ $\left[\Sigma\left(E^{\prime} \rightarrow E, \mu_{0}\right)\right]$ is not required. One needs only the first moment

$$
\Sigma_{0}\left(E^{\prime} \rightarrow E\right)=\int \Sigma\left(E^{\prime} \rightarrow E, \mu_{0}\right) d \mu_{0}
$$

and the average cosine of the scattering angle

$$
\bar{\mu}_{0}\left(E^{\prime}\right)=\frac{\int d E \int d \mu_{0} \mu_{0} \Sigma\left(E^{\prime} \rightarrow E, \mu_{0}\right)}{\Sigma\left(E^{\prime}\right)},
$$

where $\Sigma\left(E^{\prime}\right)$, the total cross section, is defined by

$$
\Sigma\left(E^{\prime}\right)=\int d E \Sigma_{0}\left(E^{\prime} \rightarrow E\right) .
$$

In this paper we derive expressions for $\Sigma_{0}$ and $\mu_{0}$ and present numerical results. The basic difficulty, which makes the muonic case qualitatively different from neutron scattering, is the large hyperfine splitting between the triplet $(S=1)$ and singlet $(S=0)$ states. Unlike in the case of ordinary hydrogen, where the hyperfine splitting has an energy only $5.9 \times 10^{-6} \mathrm{eV}(21 \mathrm{~cm})$, the hyperfine splitting in the muonic hydrogen atom is about $0.183 \mathrm{eV}$. Since the energies considered in the work described here are of the same order, the singlet-triplet transitions must be taken into account in the diffusion process.

In Sec. II we present the kinematics of this two-level system colliding with a stationary scattering center. These kinematics are inelastic in nature, because of the transitions of the projectile between the singlet and triplet states, slightly modified by induced rotational transitions in the target, which are required to conserve angular momentum. In Sec. III we compute the change in the hyperfine transition energy, taking into account the rotational energy levels of the scatterer (i.e., a hydrogen molecule). Vibrational transitions are ignored, because of their high energy. The changes induced in the scattering cross section by the thermal motion of the molecules (Doppler effect) are presented in Sec. IV. We will use these cross sections to obtain (numerical) solutions of the time-dependent Selengut-Goertzel equations in slab geometry in a subsequent paper.

\section{KINEMATICS}

The kinematics of elastic scattering has been studied extensively. 9,10 For the inelastic case, Schiff suggests that the kinematics can be obtained from the elastic case by replacing $m_{1} / m_{2}$ (where $m_{1}$ is the mass of the projectile, and $m_{2}$ the mass of the target) by

$$
\gamma=\frac{m_{1}}{m_{2}}\left(\frac{E^{\prime \prime}}{E^{\prime \prime}+Q}\right)^{1 / 2}
$$

where $E^{\prime \prime}=E^{\prime} m_{1} m_{2} /\left(m_{1}+m_{2}\right)$ is the kinetic energy of the scatterer in the center-of-mass coordinate system and $Q$ is the amount of energy that is converted from internal energy to kinetic energy during the collision process $(Q$ is positive for exothermic and negative for endothermic collisions). This substitution only partially carries out the conversion from elastic to inelastic kinematics. A complete description of the kinematics of inelastic scattering must be derived from conservation of energy and momentum, as in the elastic case. ${ }^{11}$

Since only the ratio of the projectile and the target masses is relevant for the kinematics, we may consider a projectile of unit mass and a target of mass $\boldsymbol{A}$. It is helpful to describe the collision in both the center-of-mass coordinate system (CMCS) and in the laboratory coordinate system (LCS). In LCS (respectively, CMCS), the projectile enters the collision with speed $v^{\prime}=\left(2 E^{\prime}\right)^{1 / 2}$ (respectively, $u^{\prime}$ ), scatters through the angle $\cos ^{-1} \mu_{0}$ (respectively, $\left.\cos ^{-1} \mu_{c}\right)$, and emerges with speed $v=(2 E)^{1 / 2}$ (respectively, $u$ ). Since the CMCS moves relative to the 
LCS with speed $v_{M}=v^{\prime} /(A+1)$, the speed of the target in the CMCS equals $v_{M}$ before the collision and is denoted by $v_{T}$ after the collision. Since the total momentum in the CMCS equals 0 , we have

$$
u=A v_{T} \text {. }
$$

Let $V_{i}$ and $V_{f}$ be the potential energy of the projectile (in this case, $V_{i}$ and $V_{f}$ refer to the internal energies of the triplet and singlet spin states) before and after scattering, respectively. Energy conservation in the CMCS yields

$$
\frac{1}{2}\left(v^{\prime}-v_{M}\right)^{2}+V_{i}+\frac{1}{2} A v_{M}^{2}=\frac{1}{2} u^{2}+V_{f}+\frac{1}{2} A v_{T}^{2} .
$$

Combining conservation laws (5) and (6) and noting that

$$
Q=V_{i}-V_{f}
$$

we find

$$
u=\frac{A}{A+1} \beta\left(E^{\prime}\right) v^{\prime},
$$

where

$$
\beta\left(E^{\prime}\right)=\left(1+\frac{A+1}{A} \frac{Q}{E^{\prime}}\right)^{1 / 2} .
$$

The velocity of the projectile in the LCS is the vector sum of its velocity in the CMCS and the velocity of the CMCS relative to the LCS (Fig. 1.). Using the law of cosines along with Eq. (8) yields immediately

$$
\mu_{0}=\frac{1+A \beta \mu_{c}}{\left(1+2 A \beta \mu_{c}+A^{2} \beta^{2}\right)^{1 / 2}}
$$

and a relation between the initial and final energy of the projectile in the LCS as a function of the scattering angle:

$$
\frac{E}{E^{\prime}}=\frac{1}{(A+1)^{2}}\left(A^{2} \beta^{2}+2 A \beta \mu_{c}+1\right) .
$$

As $\beta \rightarrow 1$ these reduce to the well-known equation for elastic scattering. ${ }^{5}$ At a fixed initial projectile energy $E^{\prime}$ the scattering angle in the LCS becomes a function of the final projectile energy $E$

$$
\mu_{0}(E)=\frac{1}{2}\left[(A+1)\left(\frac{E}{E^{\prime}}\right)^{1 / 2}-\frac{A^{2} \beta^{2}-1}{A+1}\left(\frac{E^{\prime}}{E}\right)^{1 / 2}\right],
$$

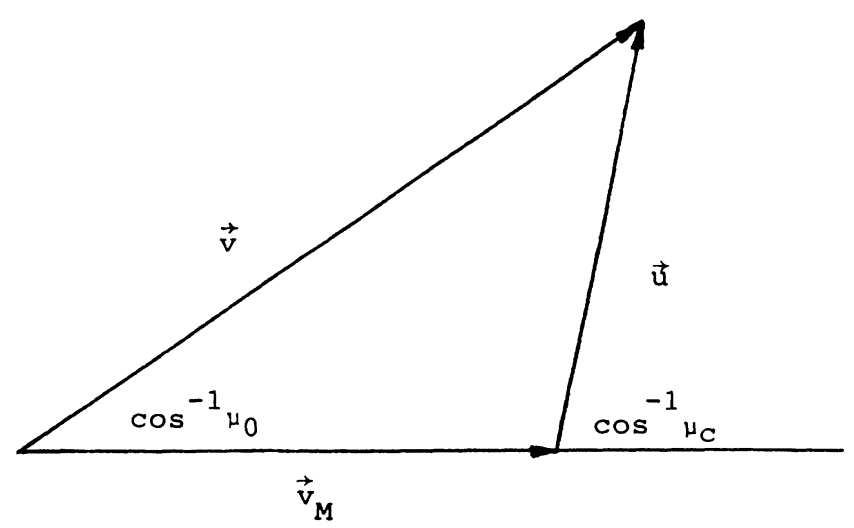

FIG. 1. Relation between the LCS and the CMCS velocities. as follows easily from (10) and (11).

Now we are ready to use the above relations in computing the scattering cross sections. Because of the low kinetic energy of the projectile, only $s$-wave (i.e., zero angular momentum) scattering is possible. Therefore the scattering is isotropic in the CMCS:

$$
\Sigma\left(E^{\prime}, \mu_{c}\right)=\frac{1}{2} \Sigma\left(E^{\prime}\right) .
$$

From

$$
\Sigma\left(E^{\prime}, \mu_{c}\right)\left|d \mu_{c}\right|=\Sigma\left(E^{\prime} \rightarrow E\right)|d E|,
$$

and Eq. (11) follows:

$$
\Sigma\left(E^{\prime} \rightarrow E, \mu_{0}\right)= \begin{cases}\frac{(A+1)^{2} \Sigma\left(E^{\prime}\right)}{4 A E^{\prime} \beta\left(E^{\prime}\right)} \delta\left(\mu_{0}-\mu_{0}(E)\right) \\ 0, & \text { otherwise, } E^{-}<E<E^{+},\end{cases}
$$

where

$$
E^{ \pm}=E^{\prime} \frac{\left[A \beta\left(E^{\prime}\right) \pm 1\right]^{2}}{(A+1)^{2}} .
$$

The $\delta$ function in Eq. (15) implements the fact that $\mu_{0}$ is uniquely determined with the choice of $E^{\prime}$ and $E$. The moments of the scattering kernel are obtained from (15) by a trivial integration. We only need the zeroth moment

$\Sigma_{0}\left(E^{\prime} \rightarrow E\right)=\left\{\begin{array}{l}\frac{(A+1)^{2} \Sigma\left(E^{\prime}\right)}{4 A E^{\prime} \beta\left(E^{\prime}\right)} \\ 0, \text { otherwise }\end{array}\right.$ if $E^{-}<E<E^{+}$

and the first moment

$$
\Sigma_{1}\left(E^{\prime} \rightarrow E\right)=\Sigma_{0}\left(E^{\prime} \rightarrow E\right) \mu_{0}(E) .
$$

The average cosine of the scattering angle in the LCS is obtained by integrating Eq. (18) over the final energy:

$$
\bar{\mu}_{0}\left(E^{\prime}\right)= \begin{cases}1-\frac{1}{2} A \beta\left(E^{\prime}\right)+\frac{1}{6} A^{2} \beta^{2}\left(E^{\prime}\right) \\ & \text { if } Q<0 \text { and } E^{\prime}<\frac{A(-Q)}{A-1} \\ \frac{2}{3 A \beta\left(E^{\prime}\right)}, & \text { otherwise . }\end{cases}
$$

Again, the well-known elastic limits of the above formulas ${ }^{11}$ are recovered by noting that $\beta \rightarrow 1$ as $Q \rightarrow 0$.

\section{EFFECTIVE INELASTIC ENERGY TRANSFER $\left(Q_{\text {eff }}\right)$}

In this section we analyze the inelastic processes $S \rightarrow T$ and $T \rightarrow S$ more fully. The first argument involves conservation of angular momentum. Because of the very low energy there is no orbital angular momentum involved. Furthermore, radiationless transitions, when allowed, are favored over photon emission by a factor of $\alpha^{2}$ ( $\alpha$ is the finite-structure constant). Therefore, the change in the 
spin of the atom must be compensated by a change in the angular momentum of the molecule. Let $I$ denote the nuclear spin ( $I=0$ corresponds to parahydrogen, $I=1$ to orthohydrogen), $J$ the rotational angular momentum of the hydrogen molecule and $K$ their sum. The Pauli principle implies that orthohydrogen is always in an odd- $J$ state and parahydrogen in an even- $J$ state.

We denote by $W_{i}$ and $W_{f}$ the initial and final rotational energy levels of the molecule and write the conservation of energy in the LCS:

$$
E^{\prime}+V_{i}+W_{i}=E+V_{f}+W_{f},
$$

where, we recall, $V_{i}$ and $V_{f}$ are the internal energies of the muonic atom. We notice that for the purposes of kinematics $V$ and $W$ play equivalent roles. Hence the proper generalization of the definition of energy transfer $Q$ [Eq. (7)] is

$$
Q_{\imath f}=V_{i}-V_{f}+W_{i}-W_{f}
$$

Since we are not interested in the scattering involving transitions between particular rotational states but only in the overall effect of all rotational transitions, we define the effective inelastic energy transfer $Q_{\text {eff }}$ as the average of $Q_{i f}$ (defined as follows).

Averaging will be carried out in two steps. In the first step we fix the initial rotational angular momentum $J=i$ and average over all possible final angular momenta $J=f$ by defining

$$
Q_{i}=\sum_{f} P_{i f} Q_{i f}
$$

where $P_{i f}$ are the conditional probabilities for ending in a final state with $J=f$, given that initially $J=i$. The possible states are constrained by conservation of energy and total angular momentum. Since at least to our knowledge the quantities $P_{i f}$ have not been calculated or measured, we approximate them and then try to argue that the result is not very sensitive to the particular approximation used. In the equal probability approximation we assume that the probabilities for all the possible final states are equal. Then $P_{i f}$ is proportional to the degeneracy of the final state, as given by its total angular momentum. More accurate is the neutron approximation, in which we take the probability for ending in a state with $J=f$ to be the same as for the case of neutron scattering and use the results of Rahman. ${ }^{12}$ The degeneracy factor is of course the same as in the equalprobability approximation.

In the second and last step we average over the thermal distribution of initial rotational angular momenta

$$
Q_{\mathrm{eff}}=\sum_{i} Q_{i} P_{i}(T)
$$

where $P_{i}$, the probability for finding the molecule in state $i$ at temperature $T$, is proportional to the Boltzmann factor and to the degeneracy of the level due to angular momentum

$$
P_{i} \sim(2 I+1)(2 J+1) \exp \left(-\frac{W_{i}}{k T}\right) .
$$

Note that $2 I+1$ equals 1 for even $J$ and 3 for odd $J$.

To understand the calculations, consider first the case $J_{i}=0$. Then, by the Pauli principle, $I=0$. Thus the total angular momentum of the molecule $K=0$. Suppose the incident atom undergoes a $S \rightarrow T$ transition. Then the final angular momentum state of the molecule must be $K=1$ to conserve angular momentum in the collision process. $K=1$ can come only as $J=1, I=1$, so in this example the molecule is excited from the $J=0$ to the $J=1$ state.

As a second example, consider an $S \rightarrow T$ transition, where initially the molecular state had $J=1$, implying $I=1, K=2,1,0$. Since the muonic atom spin $F=0$ initially, the total angular momentum $J_{T}=K$ initially. This means the molecule must be left in one of the states $K=3,2,1,0$, which can come from $J=3, I=1 ; J=2$, $I=0, I=1$; or $J=0, I=0$. All other possibilities are excluded by conservation of angular momentum and the Pauli principle.

In this second example our first average (described above) requires knowledge of the branching probabilities to each of the final states characterized by $J$. We have already noted that $J_{T}$ equals 0,1 , or 2 ; the probability of these states is $\frac{1}{9}, \frac{1}{3}$, and $\frac{5}{9}$, respectively. If $J_{T}=0$, then after the collision $K=1$, since $F=1$. This allows only one possible final state, namely $J=1, I=1$. The probability of reaching it with $J_{T}=0$ is $\frac{1}{9}$. If $J_{T}=1$, then $K=2$ 1 , or 0 and there are four equally probable final states $J=3, I=1 ; J=2, I=0 ; J=1, I=1$; and $J=0, I=0$, so the probability of reaching them through $J_{T}=1$ is $\frac{1}{12}$. For $J_{T}=2, K=3,2,1$ and the allowed final states are $J=3,2,1$ all at probability $\frac{5}{27}$. Since we only care about the final state and not about the way it was reached from the given initial state, we sum the probabilities for different $J_{T}$ yielding the same final state $J$. This tells us that the molecule goes to the state $J=3$ with probability $\frac{1}{12}+\frac{5}{27}=\frac{29}{108}$, to the state $J=2$ with the same probability, to $J=1$ with probability $\frac{1}{12}+\frac{5}{27}+\frac{1}{9}=\frac{41}{108}$, and to $J=0$ with probability $\frac{1}{12}$.

In the neutron approximation, the above probabilities are weighted with the weight of neutron cross sections obtained from Rahman, ${ }^{12}$ but numerically, there is no significant difference-see Figs. 2 and 3.

The results of the calculation for two different temperatures are presented in Figs. 2-4. At $T=100 \mathrm{~K}$, only the lowest two rotational levels were taken into account, since they represent $99 \%$ of the ambient population, while at $T=300 \mathrm{~K}$ levels up to $J=3$ contribute appreciably. Corresponding to the two inelastic processes ( $T \rightarrow S$ and $S \rightarrow T$ ), we have two curves in each figure $\left(Q_{\mathrm{eff}}^{T S}\right.$ and $Q_{\mathrm{eff}}^{S T}$, or rather $-Q_{\mathrm{eff}}^{S T}$, which is positive and hence more convenient to plot). The curve $|Q|=0.183$ $\mathrm{eV}$ is given for reference since if the rotational transitions are neglected, $Q^{T S}=|Q|$ and $Q^{\mathrm{ST}}=-|Q| \cdot Q_{\text {eff }}$ is a function of the initial kinetic energy of the muonic atom $E^{\prime}$. In the equal probability approximation this is due to the fact that at higher $E^{\prime}$ more final states are allowed by energy conservation, and so $Q_{\text {eff }}$ has steps corresponding to the opening of new channels. In addition, in the neutron approximation, the conditional probabilities $P_{i f}$ are 


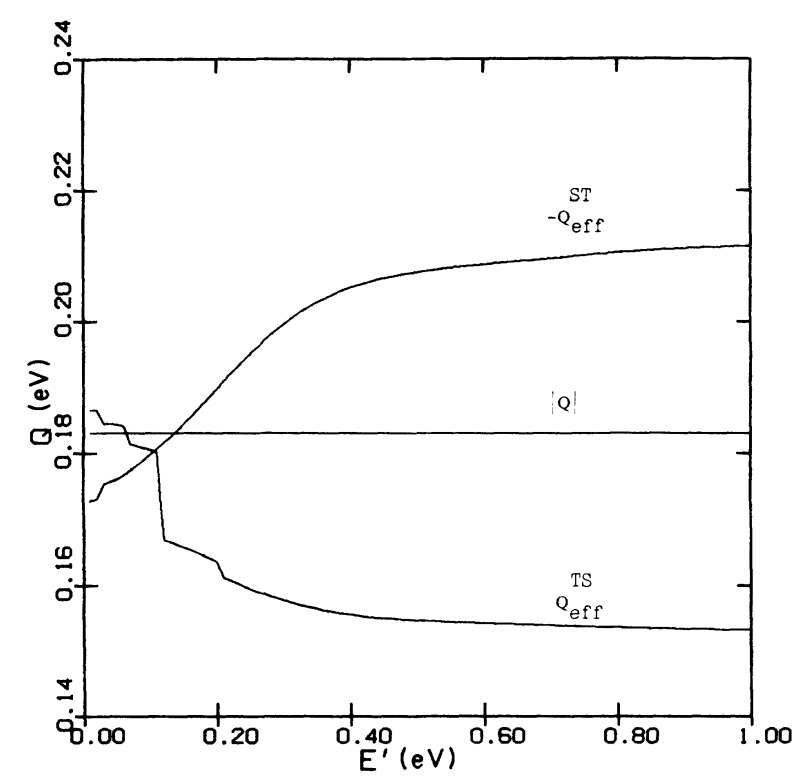

FIG. 2. Effective inelastic energy transfers $Q_{\text {eff }}^{T S}$ and $-Q_{\text {eff }}^{S T}$, as functions of the initial kinetic energy at $300 \mathrm{~K}$ (neutron approximation).

functions of $E^{\prime}$. At least qualitative agreement of the results given by the simple minded equal probability approximation and the neutron approximation shows that the result is not very sensitive to the values of $P_{i f}$. Therefore the neutron approximation should give reasonable results.

The results show that the effect of rotations is large enough that it should be taken into account, since $Q_{\text {eff }}$ represents an about $15 \%$ correction compared to $Q$.

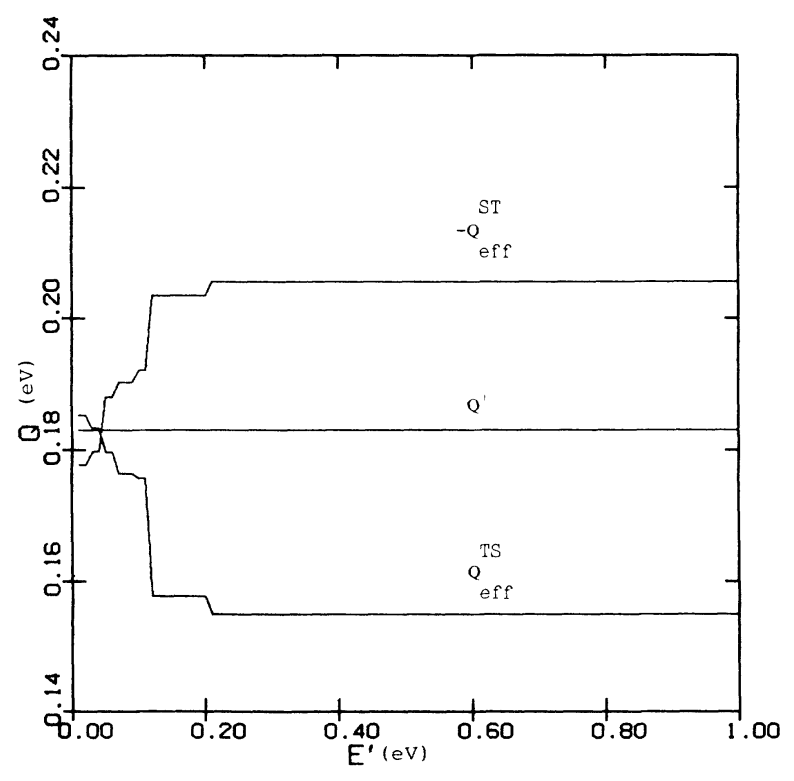

FIG. 3. Same as Fig. 2, using the equal-probability approximation.

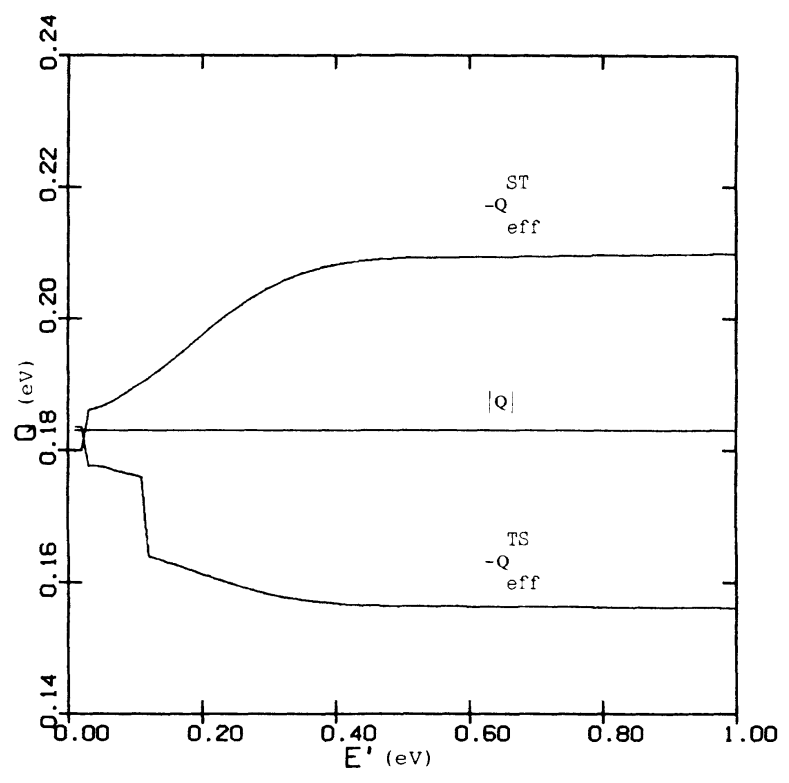

FIG. 4. Same as Fig. 2, for $100 \mathrm{~K}$.

\section{DOPPLER EFFECT}

The expressions derived for $\Sigma_{0}\left(E^{\prime} \rightarrow E\right)$ and $\mu_{0}\left(E^{\prime}\right)$ in Sec. II assume that the target molecules are at rest, i.e., apply strictly only for $T=0$. For $T>0$, the thermal motion of the molecule must be considered. The standard procedure ${ }^{13}$ is to compute $\Sigma_{0}$ and $\mu_{0}$ for a fixed molecule and to average the result over the Maxwellian distribution of these velocities.

The resulting cross section $\Sigma_{0}\left(E^{\prime} \rightarrow E ; T\right)$ was first obtained by Wigner and Wilkins ${ }^{14}$ for elastic collisions between neutrons and nuclei. This cross section can be used if it is modified in two ways, first for the inelasticity and second for the fact that the scattering centers are bound in molecules. (The available cross-section data are for collisions between muonic hydrogen atoms and ordinary hydrogen atoms, not molecules.)

The second correction has been introduced by Messi$\mathrm{ah}^{15}$ for the case of incident neutrons. As we have already explained, the muonic atoms are very similar to neutrons, so the same correction has been adopted. It is

$$
\sigma_{b}=\left(1.2+0.488 \frac{k T}{E^{\prime}}\right) \sigma_{f},
$$

where $\sigma_{f}$ is the "free" cross section, i.e., for the unbound hydrogen atom. The cross section of the hydrogen molecule is then $\sigma_{m}=2 \sigma_{b}$. Messiah's theory is based on the assumption that the initial energy of the projectile is large compared to the mean level spacing of the initial rotational states of the target molecule. ${ }^{15}$ In effect, this means we can safely apply our theory to muonic atoms whose original energy is greater than about $0.01 \mathrm{eV}$.

The first correction involves introducing $Q_{\text {eff }}$ into the Wigner-Wilkins cross section. We begin with the equation $^{13}$ 


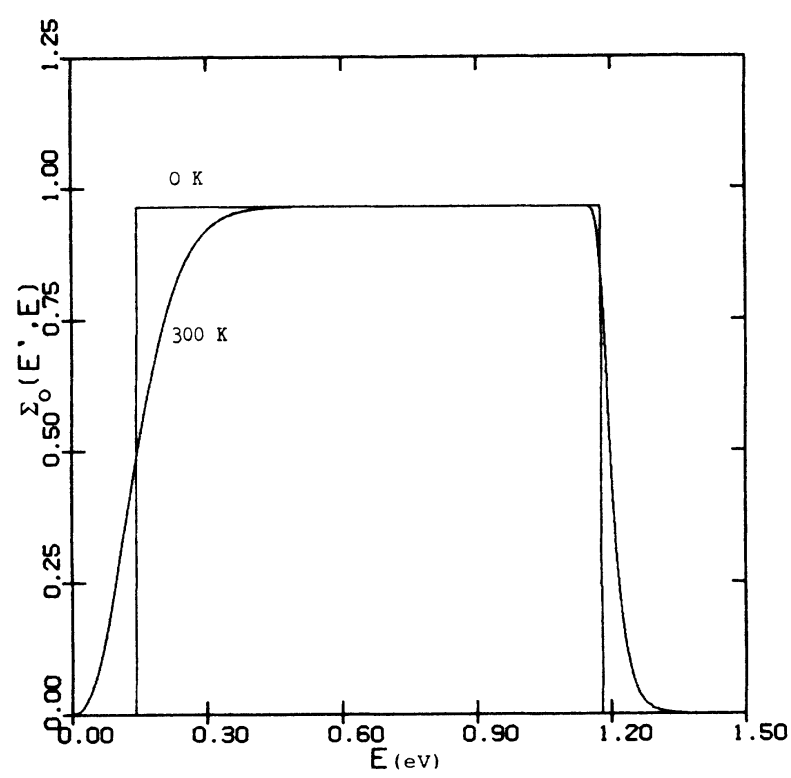

FIG. 5. The zeroth moment of the scattering cross section $\Sigma_{0}\left(E^{\prime} \rightarrow E\right)$, as a function of $E$, for $E^{\prime}=1 \mathrm{eV}$, at $300 \mathrm{~K}$ and at 0 $\mathbf{K}$.

$$
\begin{aligned}
\Sigma\left(E^{\prime}\right. & \left.\rightarrow E ; \mu_{0}\right) \\
= & \frac{N \sigma_{m}}{4 \pi}\left(\frac{E}{E^{\prime}}\right)^{1 / 2} \frac{1}{2 \pi} \\
& \times \int_{-\infty}^{\infty} d t \exp \left(-i\left(E^{\prime}-E\right) t-\frac{K^{2}}{2 A}\left(T t^{2}-i t\right)\right)
\end{aligned}
$$

Here $N$ is the number density of hydrogen molecules, $T$ is the temperature, and $K$ is the momentum transfer,

$$
\frac{1}{2} K^{2}=E^{\prime}+E-2\left(E^{\prime} E\right)^{1 / 2} \mu_{0} .
$$

Units in Eqs. (26) and (27) have been chosen such that $\hbar=1, k=1$, and the muonic atom mass equals 1 .

To adapt Eq. (26) to inelastic scattering we first multiply the integrand by a factor $\exp \left(-i Q_{\text {eff }} t\right)$ and secondly, the cross section must be renormalized to have the correct limit as $T \rightarrow 0$. This requires the replacement

$$
\sigma_{m} \rightarrow \frac{(A+1)^{2}}{\beta A^{2}} \sigma_{m}
$$

The zeroth moment of the cross section is obtained from Eq. (26) by integrating over $\mu_{0}$ and can be written in the form

$$
\begin{aligned}
& \Sigma_{0}\left(E^{\prime} \rightarrow E\right) \\
& =\frac{\Sigma\left(E^{\prime}\right)(A+1)^{2}}{8 A E^{\prime} \beta\left(E^{\prime}\right)}\left\{e^{q+\varepsilon^{\prime}-\varepsilon}[\operatorname{erf}(a)-\operatorname{erf}(b)]\right. \\
& +\operatorname{erf}(c)-\operatorname{erf}(d)\},
\end{aligned}
$$

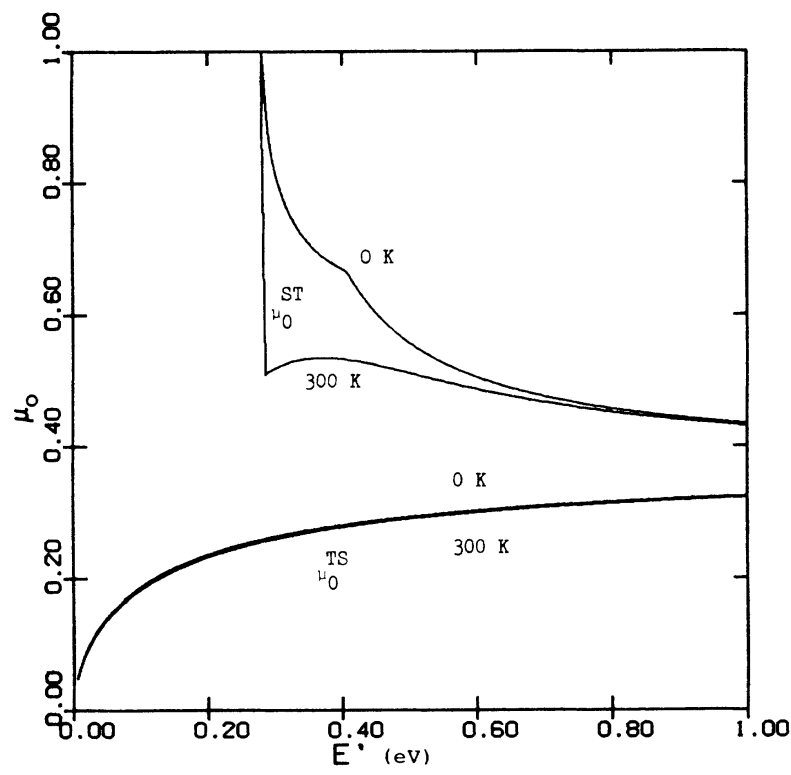

FIG. 6. The average cosines of the scattering angle $\mu_{0}^{S T}\left(E^{\prime}\right)$ and $\mu_{0}^{T S}\left(E^{\prime}\right)$, at $300 \mathrm{~K}$ and at $0 \mathrm{~K}$.

with

$$
\begin{aligned}
& \left.\begin{array}{l}
a \\
c
\end{array}\right\}=\frac{1}{2 A^{1 / 2}}\left(\varepsilon^{\prime 1 / 2}+\varepsilon^{1 / 2}\right)\left\{\begin{array}{l}
+ \\
-
\end{array}\right\} \frac{A^{1 / 2}}{2} \frac{q+\varepsilon^{\prime}-\varepsilon}{\left(\varepsilon^{1 / 2}+\varepsilon^{1 / 2}\right)}, \\
& \left.\begin{array}{l}
b \\
d
\end{array}\right\}=\frac{1}{2 A^{1 / 2}}\left|\varepsilon^{1 / 2}-\varepsilon^{1 / 2}\right|\left\{\begin{array}{l}
+ \\
-
\end{array}\right\} \frac{A^{1 / 2}}{2} \frac{q+\varepsilon^{\prime}-\varepsilon}{\left|\varepsilon^{\prime 1 / 2}-\varepsilon^{1 / 2}\right|},
\end{aligned}
$$

where

$$
q=\frac{Q}{T}, \quad \varepsilon^{\prime}=\frac{E^{\prime}}{T}, \quad \varepsilon=\frac{E}{T} .
$$

To help appreciate the importance of the Doppler correction we show in Fig. $5 \Sigma_{0}\left(E^{\prime} \rightarrow E\right)$ as a function of $E$, for $E^{\prime}=1 \mathrm{eV}$, at 300 and $0 \mathrm{~K}$. In Fig. 6 we show $\mu_{0}\left(E^{\prime}\right)$ versus $E^{\prime}$ for the same two temperatures.

\section{ACKNOWLEDGMENTS}

We are very grateful to Professor Robert Siegel for proposing this problem and would like to thank him for numerous valuable discussions. This work has been supported by the U.S. Department of Energy under Grant No. DE-FG05-87ER25033 and the National Science Foundation under Grant No. DMS 8312451.

${ }^{4}$ H. Bossy et al., Phys. Rev. Lett. 55, 1870 (1985).

5J. S. Cohen, Phys. Rev. Lett. 58, 1407 (1987).

${ }^{6}$ M. Leon and J. S. Cohen, Phys. Rev. A 31, 2680 (1985).

${ }^{7}$ M. Leon, Phys. Rev. Lett. 52, 605 (1984). 
${ }^{8}$ J. H. Ferziger and P. F. Zweifel, The Theory of Neutron Slowing Down in Nuclear Reactors (MIT Press, Cambridge, 1966).

${ }^{9}$ L. I. Schiff, Quantum Mechanics (McGraw-Hill, New York, 1949).

${ }^{10}$ P. F. Zweifel and H. Hurwitz, Jr., J. Appl. Phys. 25, 1241 (1954).

${ }^{11}$ P. F. Zweifel, Reactor Physics (McGraw-Hill, New York,
1973).

${ }^{12}$ A. Rahman, J. Nucl. Energy Part A 13, 128 (1961).

${ }^{13}$ M. M. R. Williams, The Slowing Down and Thermalization of Neutrons (North-Holland, Amsterdam, 1966).

${ }^{14}$ E. P. Wigner and J. E. Wilkins, Atomic Energy Commission Report No. AECD2275, 1944 (unpublished).

${ }^{15}$ A. M. L. Messiah, Phys. Rev. 84, 204 (1951). 